\title{
THE NUMBER OF INDEPENDENT COMPONENTS OF THE TENSORS OF GIVEN SYMMETRY TYPE
}

\author{
RICHARD H. BRUCK AND T. L. WADE
}

Let $T_{i_{1}} \cdots_{i_{p}}$ be an arbitrary covariant tensor with respect to an $n$-dimensional coordinate system, and let

$$
T_{i_{1} \cdots i_{p}}={ }_{[p]} T_{i_{1} \cdots i_{p}}+\cdots+{ }_{[\alpha]} T_{i_{1} \cdots i_{p}}+\cdots+{ }_{\left[1^{p}\right]} T_{i_{1} \cdots i_{p}}
$$

represent the decomposition ${ }^{1,2}$ of $T_{i_{1}} \ldots i_{p}$ into tensors of various symmetry types, the tensor ${ }_{[\alpha]} T_{i_{1}} \cdots i_{p}$ corresponding to the partition $[\alpha]$ of the indices $i_{1} \cdots i_{p}$. The number of independent (scalar) components of $T_{i_{1}}{\cdots i_{p}}_{p}$ is $n^{p}$; and if $c_{\alpha}$ denotes the number of components of ${ }_{[\alpha]} T_{i_{1}} \ldots i_{p}$, then

$$
n^{p}=c_{[p]}+\cdots+c_{[\alpha]}+\cdots+c_{\left[1^{p}\right]}=\sum c_{\alpha} .
$$

For $p=2,3,4$, J. A. Schouten ${ }^{3}$ has obtained expressions for the $c_{\alpha}$ 's in terms of $n$; but the difficulties of his method become great for larger values of $p$. The purpose of this paper is to present a method of obtaining $c_{\alpha}$ in terms of $n$ from the character table for the symmetric group on $p$ letters.

Associated with the immanant tensor ${ }^{2} I_{(j)}^{(i)} \equiv{ }_{[\alpha]} I_{j_{1} \ldots j_{p}}^{i_{1} \ldots i_{p}}$ we have defined the numerical invariant $r=r_{\alpha}$, the $\operatorname{rank}^{4}$ of $I_{(j)}^{(i)}$, which is the greatest integer $r$ for which the tensor

$$
I_{\left(j_{1}\right) \cdots\left(j_{r}\right)}^{\left(i_{1}\right) \cdots\left(i_{r}\right)}=\left|\begin{array}{ccc}
I_{\left(j_{1}\right)}^{\left(i_{1}\right)} & \cdots & I_{\left(j_{r}\right)}^{\left(i_{1}\right)} \\
\cdot & \cdots & \\
I_{\left(j_{1}\right)}^{\left(i_{r}\right)} & \cdots & I_{\left(j_{r}\right)}^{\left(i_{r}\right)}
\end{array}\right|
$$

does not vanish; here $\left(i_{\lambda}\right)=i_{\lambda 1} \cdots i_{\lambda p}$. For convenience, let us regard $I_{(j)}^{(i)}$, for each $(i)$, as a vector $V_{(j)}$ in $N=n^{r}$ dimensions. Then from the above definition, it is clear that exactly $r_{\alpha}$ of the $N$ vectors $V_{(j)}$ are linearly independent. Since ${ }_{[\alpha]} T_{(i)} \equiv_{[\alpha]} T_{j_{1}} \cdots_{j_{p}}$ may be defined by

$$
{ }_{[\alpha]} T_{(j)}={ }_{[\alpha]} I_{(j)}^{(l)} T_{(l)} ;
$$

Presented to the Society, November 22, 1941 under the title The number of independent components of the tensor ${ }_{[\alpha]} T_{i_{1}} \ldots i_{p}$; received by the editors November 19, 1942.

${ }^{1} \mathrm{H}$. Weyl, The classical groups, Princeton, 1939, chap. IV.

2 T. L. Wade, Tensor algebra and Young's symmetry operators, Amer. J. Math. vol. 63 (1941) pp. 645-657.

${ }^{3}$ J. A. Schouten, Der Ricci-Kalkul, Berlin, 1924, chap. VII.

${ }^{4}$ Richard H. Bruck and T. L. Wade, Bisymmetric tensor algebra, II, Amer. J. Math. vol. 64 (1942) pp. 734-753. We shall refer to this paper as B.T.A.II. 
exactly $r_{\alpha}$ of the components of $T_{(j)}$ are linearly independent; thus $c_{\alpha} \leqq r_{\alpha}$. But as an alternative way of writing equation (32), B.T.A. II,

$$
n^{p}=r_{[p]}+\cdots+r_{[\alpha]}+\cdots+r_{\left[1^{p}\right]}=\sum r_{\alpha} .
$$

Hence, using (2),

$$
n^{p}=\sum c_{\alpha} \leqq \sum r_{\alpha}=n^{p}
$$

and since the numbers $c_{\alpha}, r_{\alpha}$ are non-negative we conclude this fact.

THEOREM I. $c_{\alpha}=r_{\alpha}$.

Combining Theorem I with Theorem V, B.T.A. II, we obtain this theorem.

THEOREM II.

$$
c_{\alpha}=\frac{f_{\alpha}}{{ }_{p} !} \sum_{(\rho)} \chi_{\alpha}^{(\rho)} \cdot \nu_{\rho} n^{k \rho},
$$

where $\chi_{\alpha}^{(\rho)}$ is the characteristic for class $(\rho)$ corresponding to the irreducible representation $[\alpha]$ of the symmetric group on p letters,

$f_{\alpha}$ is the characteristic corresponding to the class $\left(1^{p}\right)$,

$\nu_{\rho}$ is the order of class $(\rho)$,

$k_{\rho}=\rho_{1}+\rho_{2}+\cdots+\rho_{p}$, where $\rho=\left(1 \rho_{1}, 2^{\rho_{2}}, \cdots, p^{\rho_{p}}\right)$.

Another method of finding $c_{\alpha}$ is given by G. de B. Robinson ${ }^{5}$ in relating $r_{\alpha}$ to A. Young's substitutional analysis.

For $p=4$ the character table is, ${ }^{6}$ with the additional row of values of $k_{\rho}$ inserted:

$\begin{array}{lccccrr}\text { Class: } & (\rho) & \left(1^{4}\right) & \left(1^{2}, 2\right) & (1,3) & (4) & \left(2^{2}\right) \\ \text { Order: } & v_{\rho} & 1 & 6 & 8 & 6 & 3 \\ & k_{\rho} & 4 & 3 & 2 & 1 & 2 \\ & {[4]} & 1 & 1 & 1 & 1 & 1 \\ & {[3,1]} & 3 & 1 & 0 & -1 & -1 \\ & {\left[2^{2}\right]} & 2 & 0 & -1 & 0 & 2 \\ & {\left[2,1^{2}\right]} & 3 & -1 & 0 & 1 & -1 \\ & {\left[1^{4}\right]} & 1 & -1 & 1 & -1 & 1\end{array}$

From this, using Theorem II, we have, for example

${ }^{5}$ G. de B. Robinson, Note on a paper by R. H. Bruck and T. L. Wade, Amer. J. Math. vol. 64 (1942) p. 753.

${ }^{6} \mathrm{D}$. E. Littlewood, Theory of group characters and matrix representations of groups, Oxford, 1940, p. 265. 


$$
\begin{aligned}
c_{[4]}= & (1 / 4 !)\left\{1 \cdot 1 \cdot n^{4}+1 \cdot 6 \cdot n^{3}+1 \cdot 8 \cdot n^{2}+1 \cdot 6 \cdot n+1 \cdot 3 \cdot n^{2}\right\} \\
& =C_{n+3,4}
\end{aligned}
$$

and

$$
\begin{aligned}
c_{[3,1]} & =(3 / 4 !)\left\{3 \cdot 1 \cdot n^{4}+1 \cdot 6 \cdot n^{3}+0 \cdot 8 \cdot n^{2}-1 \cdot 6 \cdot n-1 \cdot 3 \cdot n^{2}\right\} \\
& =9 C_{n+2,4} .
\end{aligned}
$$

In this manner we obtain the following tables of $c_{\alpha}$ :

Three-indexed tensors

$$
\begin{array}{cccc}
{[\alpha]} & {[3]} & {[2,1]} & {\left[1^{3}\right]} \\
c_{\alpha} & C_{n+2,3} & 4 C_{n+1,3} & C_{n, 3} \\
& \\
& \text { Four-indexed tensors }
\end{array}
$$

$\begin{array}{cccccc}{[\alpha]} & {[4]} & {[3,1]} & {\left[2^{2}\right]} & {\left[2,1^{2}\right]} & {\left[1^{4}\right]} \\ c_{\alpha} & C_{n+3,4} & 9 C_{n+2,4} & n C_{n+1,3} & 9 C_{n+1,4} & C_{n, 4}\end{array}$

Five-indexed tensors

$\begin{array}{cccccccc}{[\alpha]} & {[5]} & {[4,1]} & {[3,2]} & {\left[3,1^{2}\right]} & {\left[2^{2}, 1\right]} & {\left[2,1^{3}\right]} & {\left[1^{5}\right]} \\ c_{\alpha} & C_{n+4,5} & 16 C_{n+3,5} & 5 n C_{n+2,4} & 36 C_{n+2,5} & 5 n C_{n+1,4} & 16 C_{n+1,5} & C_{n, 5}\end{array}$

Six-indexed tensors
$\begin{array}{lll}{[\alpha]} & {[6]} & {[5,1} \\ c_{\alpha} & C_{n+5,6} & 25 C_{n+4,0}\end{array}$
$[4,2] \quad\left[4,1^{2}\right]$
$\left[3^{2}\right] \quad[3,2,1]$
$c_{\alpha} \quad C_{n+5,6} \quad 25 C_{n+4,6} \quad(27 n / 2) C_{n+3,5} \quad 100 C_{n+3,6} \quad(5 / 3) C_{n+2,4} C_{n+1,2} \quad(128 n / 3) C_{n+2,5}$

$\begin{array}{cccccc}{[\alpha]} & {\left[3,1^{3}\right]} & {\left[2^{3}\right]} & {\left[2^{2}, 1^{2}\right]} & {\left[2,1^{4}\right]} & {\left[1^{6}\right]} \\ c_{\alpha} & 100 C_{n+2,6} & (5 / 3) C_{n+1,4} C_{n, 2} & (27 n / 2) C_{n+1,5} & 25 C_{n+1,6} & C_{n, 6}\end{array}$

UNIVERSITY OF WISCONSIN AND

University of Alabama 